Dept. for Speech, Music and Hearing Quarterly Progress and Status Report

\title{
What happens during vocal warm-up?
}

Elliot, N. and Sundberg, J. and Gramming, P.

journal: STL-QPSR

volume: 34

number: 1

year: 1993

pages: $\quad 095-102$ 



\title{
WHAT HAPPENS DURING VOCAL WARM-UP?
}

\author{
Ninni Elliot, Johan Sundberg, \& Patricia Gramming*
}

\begin{abstract}
Most singers prefer to warm up their voices before performing. Also patients suffering from nonorganic dysphonia often tend to momentarily improve their voice function after the first voice exercises in each training session. The physiological effects underlying such improvements are largely unknown. However, it does not seem unlikely that they reflect an increased mobility of the vocal folds, possibly caused by an increased blood flow. Gramming (1988) assumed that such an increased mobility explained why the phonation threshold pressures were higher in voice patients with nonorganic dysphonia than in healthy controls. In the present investigation, the effects of warm-up exercises on the phonation threshold pressures are examined in a group of singers.
\end{abstract}

\section{INTRODUCTION}

Phonetograms, or voice range profiles, are frequently used in phoniatric practise to assess vocal function (Luchsinger, 1953; Vogelsanger, 1954; Damsté, 1970; Komiyama, Watanabe, \& Ryu, 1972; Klingholz, Martin, \& Jolk, 1985; Schutte \& Seidner, 1983; Coleman \& Mabis, 1977; Gramming, 1988). Gramming observed that the lower phonetogram contour was significantly elevated in male patients suffering from nonorganic dysphonia and returned to normal after recovery. As these differences agreed with differences in the associated subglottal pressures, Gramming speculated that they reflect changes in the mobility in the membranous portion of the vocal folds.

As vocal loudness is regulated by means of subglottal pressure, the lower contour in a phonetogram is obviously related to the lowest subglottal pressure capable of bringing the vocal folds to oscillation, the so-called phonation threshold pressure (PTP). This pressure has been the object of several studies (Baer, 1975; Finkelhor, Titze, \& Durham, 1988; Holmberg, Hillman, \& Perkell, 1989; Ishizaka \& Matsudaira, 1972; Titze 1988; Titze, 1992a and b). Based on a theoretical model of the vocal fold oscillator, Ishizaka and Matsudaira predicted that the PTP is proportional to the transglottal pressure coefficient, the viscous damping of the vocal folds, the surface wave velocity, the prephonatory glottal width, and the inverse of the vocal fold thickness. In other words, PTP can be expected to rise if the vocal folds become less mobile, if the surface wave velocity or the glottal adduction are increased and if the vocal fold thickness is decreased.

As the vocal fold thickness decreases with voice fundamental frequency, the PTP increases with pitch. This prediction has been repeatedly corroborated by measurements (e.g., Gramming, 1991). Titze (1992a) derived a theoretically based function for this dependence.

Finkelhor and collaborators studied the dependence of the PTP on the vocal fold viscosity. They manipulated this viscosity in excised canine larynges and found

${ }^{*}$ Phoniatric Department, Karolinska Hospital, Stockholm 
changes of the PTP that were in accordance with the theoretical prediction. Thus, when they reduced the viscosity, the PTP tended to drop. Verdolini-Marston, Titze, \& Druker (1990) further elaborated this experiment by inducing changes of hydration in human subjects. They found that the average PTP dropped significantly in the "wet" conditions of their six subjects, although the effect varied with pitch and between subjects. The greatest effect of decreased viscosity was observed at the highest pitch.

According to Safran, Seaber, \& Garrett (1989) warm up affects the mechanical characteristics of muscle tissue. After warm up, the muscle temperature is raised and hence the viscosity of the muscle tissue is decreased. Assuming that vocal warm up has the same effects on laryngeal musculature as on other muscles, it can be hypothesized that vocal warm up induces a lowering of the PTP. The purpose of the present investigation was to test this hypothesis in a pilot study of a group of amateur singers.

\section{EXPERIMENT}

Nine subjects participated in the experiment, six female and three male, age range 2244 and 32-57, respectively. They all had a considerable amount of voice training but were all amateur singers in the sense that they did not earn their living from singing and typically practised singing once or twice per week, mostly in choirs. The reason why we chose amateurs was that the warm up appears to typically have a greater effect on the voice in such subjects as compared to professional singers who seem to be more or less constantly warmed up, perhaps because of the frequent use of their professional voice.

Data were collected for all subjects in an anechoic chamber, and, in addition, also in a sound treated booth for three of the subjects. In ascending order they sang the pitches C, E, G, and A in each octave throughout their ranges, starting from the lowest pitch they could produce. Each pitch was given to the subjects from a synthesizer. On each pitch the subjects repeated the syllable [pa] at least 6 times, as softly as possible. They held a plastic tube, ID $4 \mathrm{~mm}$, connected to a calibrated pressure transducer (Glottal Enterprises) in their mouth corner and the subglottal pressure was captured as the oral pressure during the [p]-occlusion. To ensure a constant subglottal pressure for all syllables sang at each pitch, the subjects were instructed to sustain a tone of constant loudness interleaved with the [p] consonant, avoiding emphasis on the consonant. Whenever needed, the subjects were reminded to produce the softest possible phonation. The subjects first sang a complete series of pitches as softly as possible, and then repeated the same procedure using a degree of vocal loudness that they perceived as "comfortable". After the first recording, the subjects had a warm up session with a professional singing teacher (coauthor NE). This warm up procedure took $30 \mathrm{~min}$, approximately. Thereafter the subjects repeated the same recording procedure.

The pressure data were recorded on a TEAC multichannel FM DAT recorder with the audio signal picked up by a high fidelity microphone at a constant distance of 30 $\mathrm{cm}$ on a second channel. The analysis of the pressure data was carried out by means of an oscillograph. Mostly, the pressure data appeared as a series of peaks of similar amplitudes and with flat tops. This suggested that the subjects managed to keep a constant subglottal pressure for each pitch. However, some of the soprano subjects tended to produce edged peaks a their top pitches, possibly because of a particular 
articulatory behavior acquired during training. All such data were discarded, as they could be assumed not to reflect subglottal pressure accurately (Holmberg, 1993). For this reason, the data from two of the female subjects had to be eliminated from further analysis.

\section{RESULTS}

All subjects reported that the warm-up session was successful, so that they felt the voice to be in better condition during the second part of the experiment. However, one of the subjects (coauthor JS) made the experiment about a week after recovery from a laryngitis. In this case, the warm-up session did not bring his voice to what he conceived as typical for a warmed-up condition, his pitch range being extended a few semitones below his normal lower limit after the warm-up thus suggesting an atypical vocal fold status. He repeated the experiment three times, thus providing comparative data.

Figure 1 shows the PTP data from his three repetitions of the experiment together with Titze's (1992) theoretically derived PTP values. Two of the subject's curves are rather similar, the values generally agreeing within $\pm 0.5 \mathrm{~cm}$ of $\mathrm{H}_{2} \mathrm{O}$, although somewhat greater discrepancies occurred at high fundamental frequencies. These curves are in good accordance with the theoretical curve, except in the lowest pitch range. One of the subject's curves consistently showed higher values both before and after the warm up. These data pertain to the subject's post cold session. These results suggest that the data obtained were reasonably reproducible and support the assumption that the data reflect vocal fold status.

BEFORE

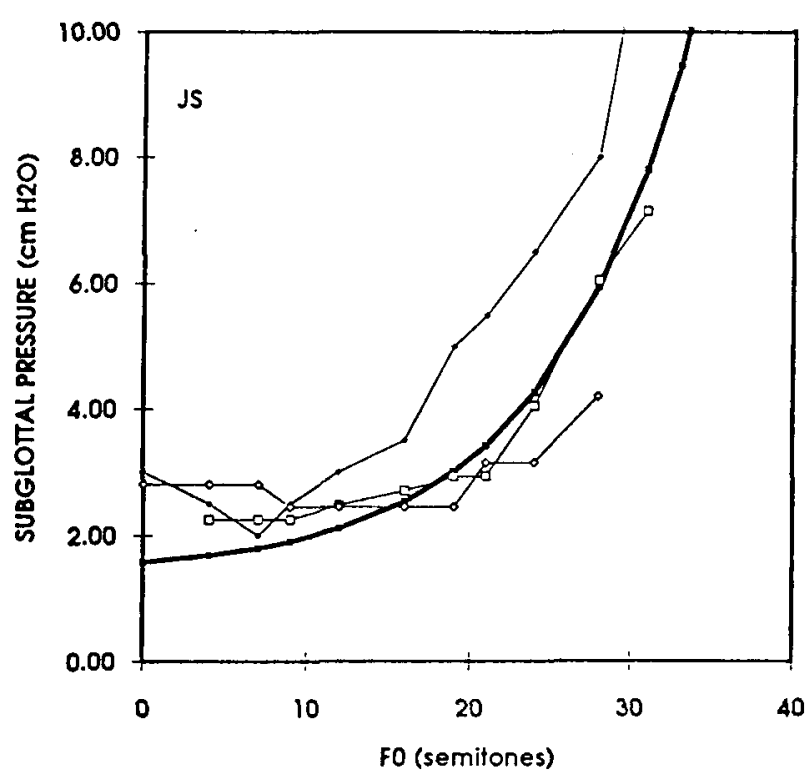

AFTER

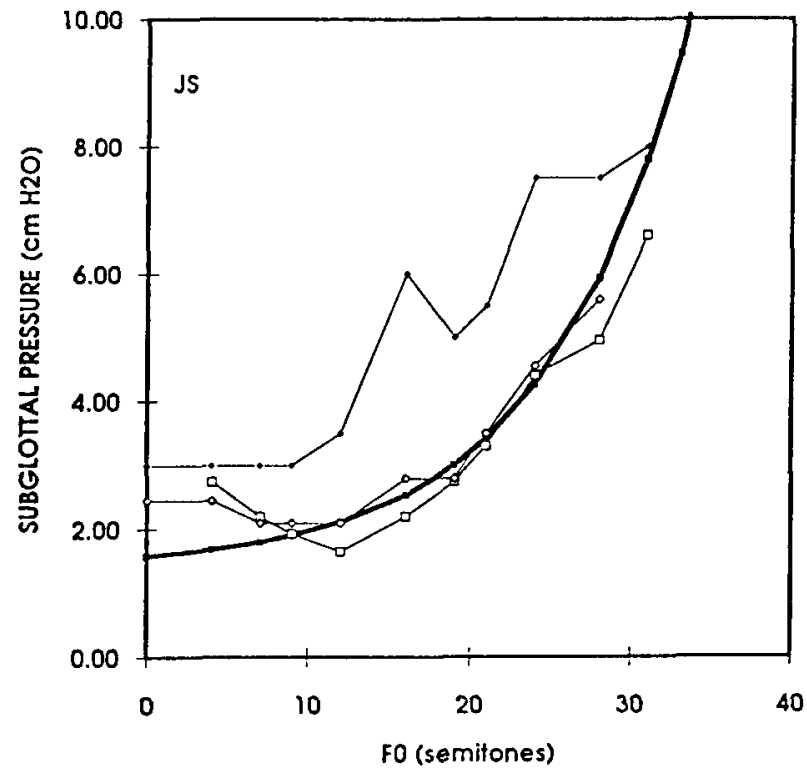

Fig. 1. Phonation threshold pressures up for a male subject before and after vocal warm-up (left and right panels) as function of voice fundamental frequency expressed in semitones relative to the indicated pitch reference. Symbols refer to different experimental sessions; filled diamonds refer to the occasion when the subject was recovering from an acute laryngitis. The heavy curve represents Titze's theoretically derived prediction of the phonation threshold pressure. 
Figure 2 presents the corresponding PTP values for all subjects before the warmup. Again, Titze's theoretically predicted curve is shown for comparison. The data for the female subjects show a great interindividual scatter. One of the subjects had PTP values that were mostly at least about $2 \mathrm{~cm} \mathrm{H}_{2} \mathrm{O}$ above the group average and another subject showed exceedingly low values apparently void of pitch dependence. The interindividual differences among the male subjects was smaller, the data approximating the theoretical curve rather closely. We conclude that our PTP data are likely to represent reliable information.

PTP FEMALE SUBJECTS, PRAE

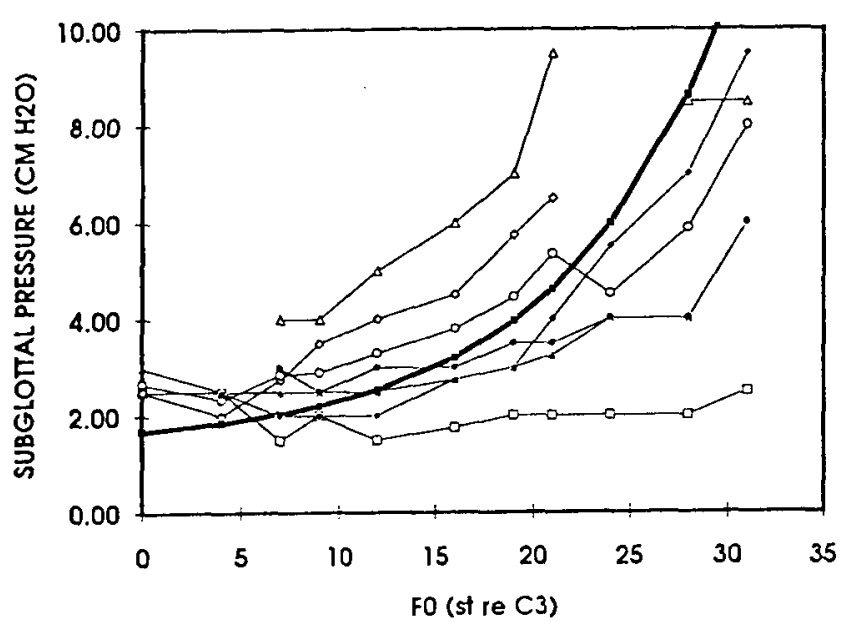

PTP MALE SUBJECTS, PRAE

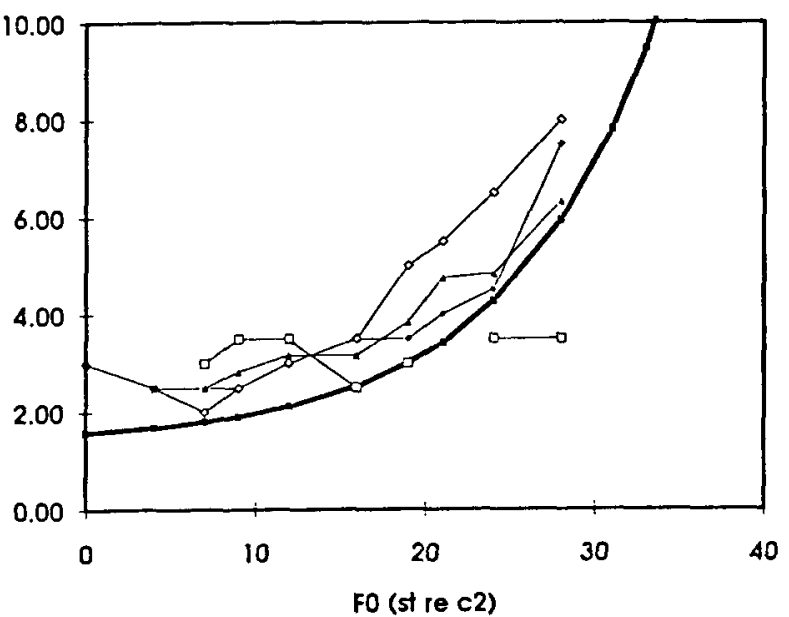

Fig. 2. Phonation threshold pressures before vocal warm-up for the female and male subjects (left and right panels) as function of voice fundamental frequency expressed in semitones relative to the indicated pitch references. Symbols refer to subjects. The heavy curve represents Titze's theoretically derived prediction.

To examine the effect of vocal warm up on the PTP, the ratio was computed between PTP after and before the warm-up procedure. The data for the entire group of subjects are shown in the two panels of Fig. 3, the left showing data for female and the right for male subjects. The heavy curve represents the average. The interindividual variability in the female group is considerable, the values varying between 0.5 and 1.5. However, for most subjects the ratio showed a clear though individual pitch dependence. For example, for one of the male subjects, the ratio consistently increased with pitch while for one of the female subjects, it consistently decreased with pitch. In another of the female subjects, it remained in the vicinity of 1.3 throughout most of her pitch range approaching 1.0 for her top pitches. The heavy average curve represents the group means. It is close to the horizontal unity line for the female subjects and mostly somewhat above this line for the male subjects.

Summarizing, we found a great variability with respect to the effect of vocal warm-up on the PTP. This pressure increased with pitch in some subjects, decreased in others, and remained basically unaffected by pitch in yet other subjects. No clear difference was found between female and male subjects. 

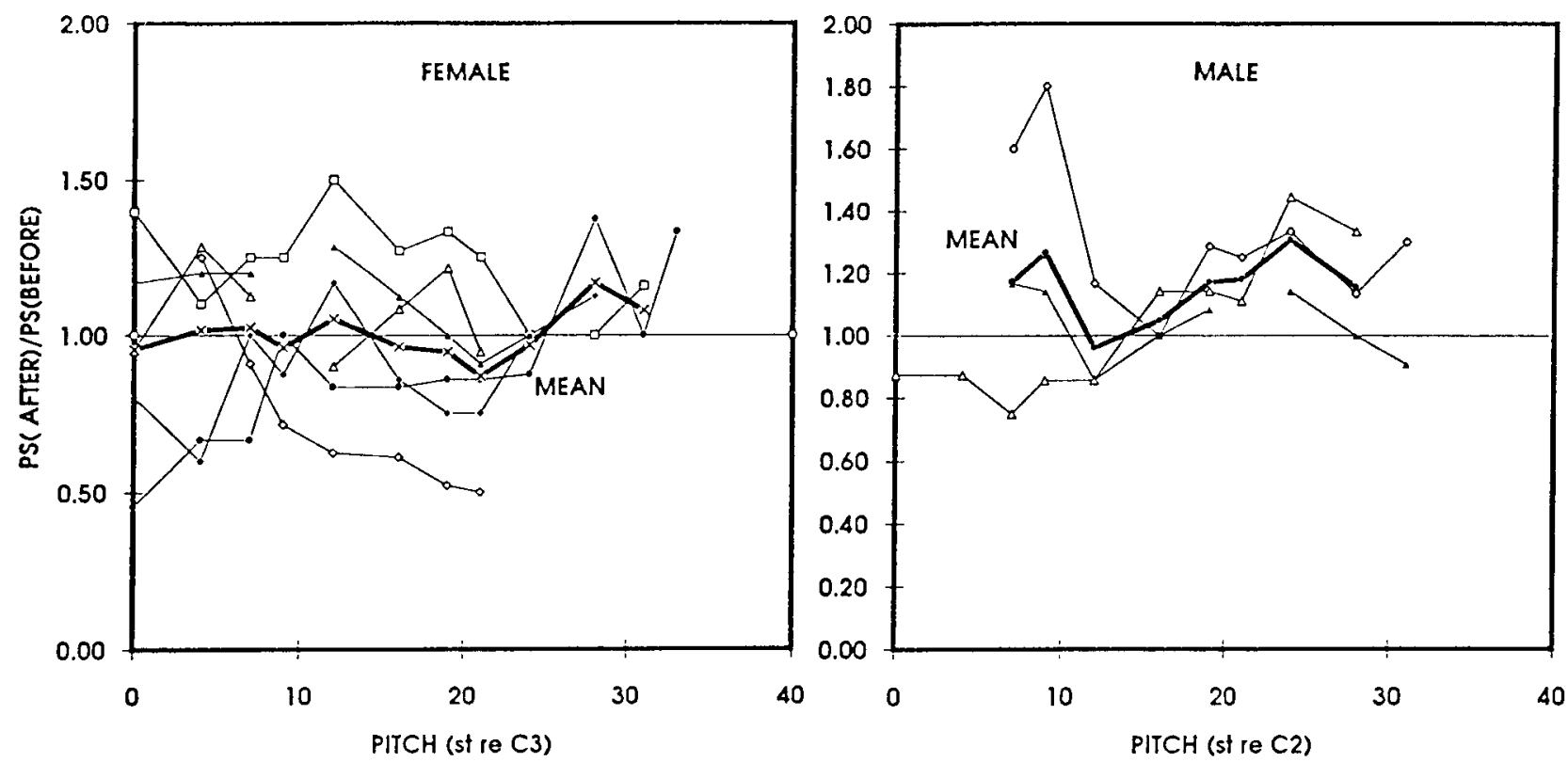

Fig. 3. Subglottal pressure ratio after and before vocal warm-up in female and male subjects as function of voice fundamental frequency expressed in semitones relative to the indicated pitch references (left and right panels, respectively). Symbols refer to subjects. The heavy line represents the group averages.

Adopting the idea of Titze, the subjects'pressures at the loudness level, which they perceived as "comfortable", were related to the PTP. The resulting pressure ratios are shown in Fig. 4. Among the female subjects one showed exceptionally high ratios. The reason was that her PTP remained low and constant throughout her range, while at her comfortable voice level, she increased her subglottal pressure with pitch. Disregarding this subject, the typical pressure ratio used for the comfortable vocal loudness remained at slightly below twice the PTP throughout the pitch range both before and after warm-up. The same applies to the male subjects.

\section{DISCUSSION}

Many subjects showed a clear pitch dependence of the warm-up effect. Only in few subjects we did find a PTP that was affected to the same extent throughout the subject's entire pitch range. However, a comparable variability in pitch dependence was found by Verdolini-Marston \& al., in their investigation of PTP shifts induced by hydration and dehydration. Only in rare cases did the results for intermediate pitches in that investigation show a coherence with those observed at low and high pitches.

The aim of the present investigation was to test the hypothesis that vocal warm-up induces a lowering of the PTP. We assumed that vocal warm-up has the same effects on laryngeal musculature as on other muscles, i.e., a rise of the temperature resulting in a lowering of the viscosity. The results showed a great inter-individual variability. What may have caused this variability?

One possibility is that the effects of vocal warm-up is not equivalent to that of a warm-up other muscles. However, this does not seem likely. First, all subjects reported that they felt in good and improved vocal shape after the warm-up session. 
Second, the vocalis muscle is participating in the vocal fold vibrations and active in control of pitch. Hence, it is vibrated and also it alternately contracts and is stretched during singing exercises. It is hard to doubt that this muscle is subject to a warm-up effect similar to that in other muscles. In other words, we see no reasons to doubt that the vocalis muscle possessed a lower viscosity after vocal warm-up in our experiment.
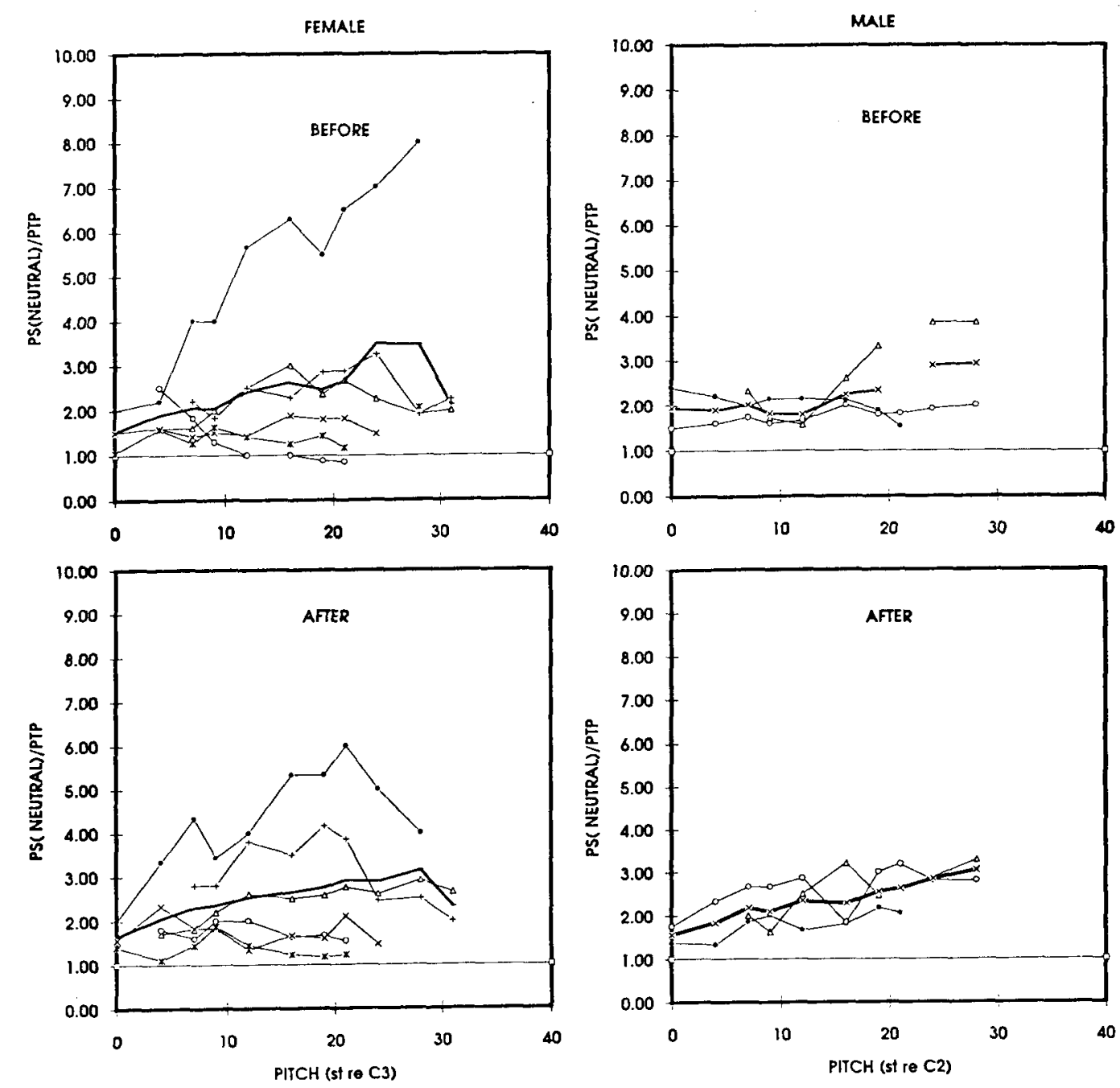

Fig. 4. Ratio of the subglottal pressure between softest and "comfortable" vocal loudness as function of voice fundamental frequency expressed in semitones relative to the indicated pitch references for female and male subjects (left and right panels, respectively) before and after vocal warm-up (upper and lower panels, respectively)

Our results suggest that the reduced viscosity in the vocalis muscle did not consistently induce a lower PTP. The PTP is, however, dependent upon a number of other factors as well, which might be influenced by a vocal warm-up. A decrease of the vocal fold thickness leads to an increase of PTP, but it is not obvious why a vocal warm-up should result in a reduced vocal fold thickness. Other factors are the surface wave velocity and the prephonatory glottal width. Also in these respects, an obvious effect of vocal warm-up is hard to see. Still, as there are several factors of relevance to the PTP, our results seem to demonstrate that the reduced viscosity was not a dominating factor in all our subjects. It may be of relevance, that the vibrational involvement of the vocalis muscle is minute in the softest possible phonation; rather, 
the vibrations seem to be limited to the surface layer mainly. According to Hirano (1981), each of the various layers of the vocal folds has different mechanical properties.

Our PTP data were in good agreement with and thus supported Titze's theoretically derived prediction, even though the PTP seems to typically increase slightly towards the lowest part of the pitch range. This increase was probably the reason why Holmberg, \& al. (1989) found that subglottal pressure in their subjects was higher not only for high pitches but also for low pitches as compared to the individual subject's neutral speaking pitch.

Many subjects experience the effects of vocal warm-up as rather physical. The instrument is felt to function in a better way. In view of this, it is quite interesting to note the great inter-individual variability which seems to exist with respect to the physical correlates of a warm-up. The variability suggests that the mechanical properties of the vocal fold differs significantly between individuals. Such a variability is likely to produce voice timbre differences.

The tendency of our subjects to use a constant factor for the PTP when singing at a comfortable vocal loudness strongly supports the idea of Titze (1992a) that subglottal pressure should be related to the PTP. In future experiments we plan to induce changes of the mechanical properties of the mucosa by medication of patients suffering from acute laryngitis. It would also be interesting to analyze the shifts in the PTP caused by starting the voice with a warm-up as compared with a vocally demanding song. It may be assumed that in the latter case remaining muscular tonus will be created in the vocal folds inflicting on the vocal fold viscosity and, thus, perhaps preventing the singer's access to pitches which demand a great vibrating mass.

As all subjects felt a clear difference in their voice function after the warm-up, and as the concomitant changes in PTP differed between subjects, the physical essence of the warm-up does not seem to dwell in the effects on the PTP. A warm-up can be assumed to be associated with many other effects. For example, the amplitudes of the higher voice source partials may increase for a given subglottal pressure, or the periodicity of the vocal fold vibrations may increase. Furthermore, though not participating in vibration, extrinsic laryngeal muscles such as the lateral cricoarytenoid may indirectly affect vibration by tuning the vibratory conditions. Also, a vocal warm-up may affect the nervous control system of phonation and optimize the vibratory conditions, e.g., the mutual tuning of subglottal pressure, vocal fold longitudinal tension, and the force of glottal adduction (Ewert Knutsson, personal communication). Such effects would be worthwhile to examine in future research.

\section{CONCLUSIONS}

Clear effects were observed of vocal warm-up on the PTP. However, they differed from subject to subject. This suggests that the decrease of the vocal fold viscosity, which presumably accompanies a vocal warm-up, is not a dominating factor for the PTP in all subjects.

\section{REFERENCES}

Baer, T. (1975): Investigation of Phonation using Excised Larynges, Diss., MIT, Cambridge, MA.

Coleman, R. \& Mabis, J. (1977): "Fundamental frequency - sound pressure level profiles of adult male and female voices," J.Speech \& Hear.Res. 20, pp. 197-204. 
Damsté, H. (1970): "The phonetogram," Practica Oto-Rhino-Laryngol. 32, pp. 185-187.

Finkelhor, B., Titze, I., \& Durham, P. (1988): "The effect of viscosity changes in the vocal folds on the range of oscillation," J.Voice 1, pp. 320-325.

Gramming, P. (1988): The Phonetogram, An Experimental and Clinical Study, Diss., Lund University.

Gramming, P. (1991): "Vocal loudness and frequency capabilities of the voice," J.Voice 5, pp. 144-157.

Hirano, M. (1981): Clinical Examination of Voice, Springer Verlag, New York.

Holmberg, E. (1980): "Laryngeal airway resistance as a function of phonation type," pp. 44-57 in (J. Lubker, Ed.) Phonetic Experimental Research, Institute of Linguistics (PERILUS II), Stockholm University.

Ishizaka, K. \& Matsudaira, M. (1972): "Fluid mechanical considerations of vocal cord vibration," Monograph 8, Speech Comm. Research Lab., Santa Barbara, CA

Klingholz, F., Martin, F., \& Jolk, A. (1985): "Die Bestimmung der Registerbrüche aus dem Stimmfeld," Sprache Stimme Gehör 9, pp. 109-111.

Komiyama, S., Watanabe, H., \& Ryu, S. (1984): "Phonetographic relationship between pitch and intensity of the human voice," Fol.Phoniat. 36, pp. 1-7.

Luchsinger, R. (1953): "Physiologie der Stimme," Fol.Phoniat. 5, pp. 58-127.

Safran, M., Seaber, A., \& Garrett, W. (1989): "Warm-up and muscular injury prevention. An update," Sports Medicine 8, pp. 239-249.

Schutte, H. \& Seidner, W. (1983): "Recommendation by the Union of European Phoniatricians: Standardizing voice area measurement - Phonetography," Fol.Phoniat. 35, pp. 286288.

Titze, I. (1992a): "Phonation threshold pressure: A missing link in glottal aerodynamics," J.Acoust.Soc.Am. 91, pp. 2926-2935.

Titze, I. (1992b): "Acoustic interpretation of the voice range profile (phonetogram)," J.Speech \& Hear.Res. 35, pp. 21-34.

Verdolini-Marston, K., Titze, I., \& Druker, D. (1990): "Change in phonation threshold pressure with induced conditions of hydration," J.Voice 4, pp. 142-151.

Vogelsanger, G. (1954): "Experimentelle Prüfung der Stimmleistung beim Singen," Fol.Phoniat. 6, pp. 193-227. 